\title{
Insegurança alimentar e sua associação com consumo de alimentos regionais brasileiros
}

\author{
Food insecurity and its association with \\ consumption of Brazilian \\ regional foods
}

Stefanie Eugênia dos Anjos Campos COELHO'

Muriel Bauermann GUBERT ${ }^{1}$

\section{R E S U M O}

\section{Objetivo}

Verificar se existem diferenças no consumo de alimentos regionais entre adolescentes em situação de insegurança alimentar quando comparados aos seguros.

\section{Métodos}

Estudo transversal realizado com adolescentes estudantes do $9^{\circ}$ ano das 26 capitais brasileiras e Distrito Federal em 2011/2012. A amostra foi composta por 15084 jovens. O consumo de alimentos regionais foi avaliado por meio de imagens. O participante deveria identificar o alimento, referindo seu consumo ou não. A situação de segurança alimentar foi aferida através da Escala Curta de Insegurança Alimentar, adaptada da escala americana e validada para o público adolescente brasileiro.

\section{Resultados}

Foram analisados os dados de 14690 adolescentes com média de idade de 14,4 anos, predominantemente do sexo feminino $(55,7 \%)$ e alunos de escolas públicas $(78,2 \%)$. Apenas 3,1\% das mães eram analfabetas. A insegurança alimentar foi mais prevalente nos domicílios com jovens do sexo masculino, que estudavam em escolas púbicas, filhos de mães analfabetas e residentes na região Norte. O consumo de hortaliças e frutas foi maior entre adolescente residentes em domićlios em insegurança alimentar e insegurança alimentar grave do que entre os seguros, em todas as regiões geográficas brasileiras. As preparações regionais são mais consumidas por jovens que vivem em domicílios seguros.

\footnotetext{
1 Universidade de Brasília, Departamento de Nutrição, Programa de Pós-Graduação em Nutrição Humana. Campus Darcy Ribeiro, Asa Norte, 70910-900, Brasília, DF, Brasil. Correspondência para/Correspondence to: MB GUBERT. E-mail: $<$ murielgubert@gmail.com>.
} 


\section{Conclusão}

Observou-se que a insegurança alimentar está associada ao maior consumo de alimentos marcadores de dieta saudável, como frutas e hortaliças regionais. A produção e consumo de alimentos regionais deve ser estimulada e valorizada como forma de promoção da alimentação saudável e de garantia da segurança alimentar e nutricional.

Palavras-chave: Adolescente. Consumo de alimentos. Segurança alimentar e nutricional.

\section{A B S T R A C T}

\section{Objective}

The aim of this study is to investigate whether the consumption of regional food among Brazilian adolescents is associated with household food security status.

\section{Methods}

This is a cross-sectional study that includes a sample of 15,084 adolescents, ninth-grade students in 26 Brazilian large cities in 2011/2012. The consumption of regional foods was evaluated using various food images; the adolescents were asked to identify the food and indicate whether they consumed that food. Household food security status was evaluated using a Short Form of the Food Insecurity Scale, adapted from the American Scale and validated for Brazilian adolescents.

\section{Results}

Data of 14,690 adolescents with a mean of the age was 14.4 years were analyzed. The majority of the adolescents were female (55.7\%) and public school students (78.2\%). Only 3.1\% of mothers were illiterate. Food insecurity was more prevalent among households with male students who were enrolled in public schools and whose mothers with were illiterate and lived in the Northern region of the country. The consumption of fruits and vegetables was higher among adolescents living in households with moderate and severe food insecurity than among those living in households that are food secure in all regions investigated. Home prepared regional foods are more commonly consumed by adolescents who live in households that are food secure.

\section{Conclusion}

It was found that food insecurity is associated with greater consumption of foods that are part of a healthy diet, such as regional fruits and vegetables. The production and consumption of regional foods should be encouraged as a way to instill healthy eating habits and ensure food security.

Keywords: Adolescent. Food consumption. Food security and nutrition.

\section{N T R O D U Ç Ã O}

A fase da adolescência é acompanhada de diversas mudanças biológicas, psicológicas, cognitivas e sociais, as quais refletem no comportamento alimentar. Por isso torna-se necessário considerar os fatores externos que influenciam a dieta nessa faixa etária, dentre eles a família e suas características, a atitude dos pais e amigos, as normas e valores sociais e culturais, a mídia, o conhecimento de nutrição e as preferências alimentares ${ }^{1}$.

O consumo alimentar dos adolescentes tem sido marcado pelo excesso de alimentos ricos em sal, gordura e açúcar - como refrigerantes, biscoitos recheados e alimentos industrializados em geral -, além da incorporação de junk foods. O consumo de frutas e hortaliças é reduzido e é comum a omissão do café da manhã, bem como a adoção de dietas monótonas e modismos alimentares ${ }^{2-4}$. Como consequência desses maus hábitos, no Brasil aproximadamente um quinto dos adolescentes está com excesso de peso.

Essa situação, de consumo de alimentos nutricionalmente inadequados e de alta prevalência de excesso de peso, evidencia uma circunstância de Insegurança Alimentar e de violação ao Direito Humano à Alimentação Adequada (DHAA), caracterizados pela falta de acesso a uma alimentação adequada e variada, em quantidade 
suficiente para a promoção de uma vida saudável $^{5}$. Tal fato pode ocorrer devido à falta de acesso financeiro/físico ao alimento ou falta de informação/proteção ${ }^{2,5}$. A insegurança alimentar, por sua vez, pode ter reflexos no desenvolvimento pleno dos adolescentes, podendo comprometer sua saúde, com repercussões inclusive na fase adulta ${ }^{6,7}$

A garantia do Direito Humano à Alimentação Adequada implica em adoção de práticas alimentares promotoras de saúde e que respeitem a diversidade cultural, além de serem ambientalmente sustentáveis ${ }^{5}$. Logo, se torna importante o incentivo à alimentação saudável baseada nos valores culturais brasileiros, com estímulo ao consumo de alimentos saudáveis - em especial os regionalmente tradicionais -, levando em consideração os aspectos comportamentais e afetivos relacionados à alimentação $0^{8,9}$.

Nesse contexto, torna-se importante conhecer o consumo alimentar do adolescente e os aspectos que o influenciam, verificando se os princípios da segurança e soberania alimentar estão sendo respeitados em seus domicílios ou comunidades. No Brasil, a literatura disponível sobre a presença de alimentos regionais na alimentação habitual dos adolescentes ainda é escassa, assim como a sua relação com a insegurança alimentar. Levando-se em consideração que o conhecimento acerca da identidade cultural da população é importante para a compreensão do aspecto sociocultural da segurança alimentar e nutricional, podendo influenciar o olhar dos profissionais de saúde, o presente estudo pretende verificar se existem diferenças no consumo de alimentos regionais entre adolescentes em situação de insegurança alimentar quando comparados aos seguros, contribuindo para a implementação e o acompanhamento de políticas sociais neste âmbito 9 .

\section{M É T O D OS}

Os dados do presente estudo são provenientes da pesquisa "Mapeamento da cultura alimentar da população adolescente nas capitais brasileiras e Distrito Federal" de caráter transversal, realizada com adolescentes estudantes de $9^{\circ}$ ano das 26 capitais brasileiras e Distrito Federal, nos anos de 2011/2012.

O processo de amostragem foi por conglomerados, com sorteio das escolas em um único estágio, de forma a gerar dados representativos para a população adolescente residente na área abrangida. Utilizou-se nível de $95 \%$ de confiança e erro aceitável de cinco pontos percentuais, tendo sido acrescentados $10 \%$ para possíveis perdas e recusas.

O número de estudantes pesquisados foi calculado com base no total de adolescentes do Brasil e em cada uma das cidades estudadas, sendo representativo para cada um destes estratos, além de ter representatividade para escolas públicas e privadas. As escolas que informaram possuir turmas de $9^{\circ}$ ano do Ensino Fundamental e laboratório de informática no censo escolar de 2010 foram ordenadas em uma lista por sorteio aleatório. A partir dessa lista as escolas eram convidadas a participar da pesquisa, uma a uma, até que se alcançasse o número de alunos estimado para cada capital. A amostra final foi composta por 15084 adolescentes, em 281 escolas.

A coleta de dados ocorreu entre setembro de 2011 e novembro de 2012, por meio de questionário online com perguntas que informavam as condições socioeconômicas e demográficas, o consumo de frutas, hortaliças e preparações regionais e a situação de segurança alimentar domiciliar. Os alunos responderam ao questionário no laboratório de informática da escola em dia letivo previamente agendado com a equipe. O controle de dados era realizado por senhas individuais fornecidas aos professores/escolas pela equipe de pesquisa, as quais os alunos tinham acesso apenas na hora de preencher o questionário. Essa medida visava evitar que estudantes de outras séries respondessem à pesquisa, bem como a dupla resposta.

O consumo de preparações e de alimentos regionais foi avaliado por imagens e perguntas, 
sendo que o adolescente deveria, após visualizar o alimento ou preparação, referir seu consumo ou não, bem como a frequência deste na época de sazonalidade do alimento na região (diário, semanal, quinzenal, mensal, anual, não consumiu). Para o presente estudo foi utilizada apenas a pergunta "consumiu" e desconsiderada a sua frequência.

A seleção das preparações regionais foi baseada no estudo de Ginani ${ }^{10}$. Foi confeccionado, então, um banco de alimentos, sendo: 13 nacionais, 27 da região Norte, 18 da Nordeste, 12 da Sul, 24 da Sudeste e 15 da região Centro-Oeste. Os alimentos foram apresentados aos alunos segundo a região de moradia e cada aluno respondeu perguntas referentes a até 15 alimentos, sorteados aleatoriamente pelo computador, incluindo os nacionais e os da sua região. Optou-se por limitar a apresentação de apenas 15 alimentos para que o questionário não ficasse muito longo, sendo, por isso, diferentes os " $n$ " amostrais para cada alimento.

Os alimentos regionais foram separados para análise nos seguintes subgrupos: hortaliças, frutas e preparações. Para a apresentação dos resultados referentes às preparações regionais, devido ao número elevado das mesmas, optou-se por utilizar neste estudo apenas as dez com maior consumo referenciado pelos adolescentes.

A situação de insegurança alimentar foi aferida através da Escala Curta de Insegurança Alimentar, adaptada da escala americana e validada para o público adolescente brasileiro ${ }^{11,12}$. A Escala Curta de Insegurança Alimentar contém 5 questões referentes à alimentação no domicílio nos últimos 12 meses, abrangendo situações desde a inexixtência de uma alimentação variada até a ocorrência de fome por falta de recursos financeiros. Os domicílios foram classificados conforme escore gerado com as respostas afirmativas sendo: entre 1 e 2 pontos, insegurança alimentar leve, entre 3 e 4, insegurança alimentar moderada, e 5 pontos, insegurança alimentar grave. Foram considerados seguros os domicílios em que o adolescente não pontuou na escala.
Para a análise dos dados foi utilizado o programa Statistical Package for the Social Sciences (SPSS Inc., Chigaco, Ilinois, Estados Unidos) para Windows ${ }^{\circledR}$ versão 17 , onde foram realizadas as análises de prevalência do consumo de frutas, hortaliças e preparações regionais por adolescentes e a sua associação com a situação de segurança alimentar domiciliar. Para melhor organização dos dados optou-se por apresentar nos resultados apenas as dez preparações mais consumidas de cada região. As prevalências de consumo foram também apresentadas para adolescentes que residiam em domicílios seguros e inseguros (abrangendo os três níveis de insegurança alimentar). As associações foram analisadas pelo teste Qui-quadrado, e foi calculada a razão de prevalência entre domicílios seguros e inseguros graves (pois nestes há presença de fome), no intuito de avaliar os dois extremos da situação de segurança alimentar domiciliar. Foram consideradas significativas associações com $p<0,05$.

A pesquisa foi aprovada pelo Comitê de Ética em Pesquisa da Universidade de Brasília ( $n^{\circ} 034 / 11$ ). As instituições de ensino convidadas consentiram com a realização da pesquisa por meio do Termo de Ciência Institucional; também foi encaminhado aos pais ou responsáveis pelos alunos participantes o Termo de Consentimento Livre e Esclarecido.

\section{RES U LT A D O S}

Após a exclusão dos casos de não resposta para um ou mais itens da escala de insegurança alimentar (394 casos), a amostra final para esta pesquisa foi de 14690 adolescentes residentes nas capitais brasileiras e Distrito Federal. A maioria era do sexo feminino $(55,7 \%)$, com média de idade de 14,4 anos (Desvio-Padrão-DP=1,15). A maior parte dos alunos participantes era de escolas públicas (78,2\%). Em relação à escolaridade materna, aproximadamente $37,0 \%$ das mães tinham ou estavam cursando o ensino superior $(29,4$ e $5,1 \%$, respectivamente) e apenas 3,1\% eram analfabetas. A insegurança alimentar foi 
mais prevalente nos domicílios com estudantes do sexo masculino, de escolas públicas, filhos de mães com baixa escolaridade e residentes na região Norte (Tabela 1).

Em relação ao consumo das hortaliças nacionais, a mandioca foi a mais consumida pelos adolescentes brasileiros $(83,1 \%)$ com uma pequena, porém significativa, diferença entre os domicílios seguros e inseguros. Esse mesmo comportamento ocorreu com as outras hortaliças nacionais pesquisadas (Tabela 2). A batata doce merece destaque, sendo a segunda mais consumida, apresentando consumo 32,0\% maior entre adolescentes com insegurança alimentar grave quando comparados com os que vivem em domicílios seguros.

Em geral, o consumo das hortaliças de acordo com a região foi maior entre adolescentes em situação de insegurança alimentar e insegurança alimentar grave do que entre os seguros, sendo estas diferenças significativas. As exceções foram o jambu e a serralha na região Norte e a mostarda e a taioba no Sudeste, mais consumidos em domicílios seguros (Tabela 2).

A gueroba apresentou consumo elevado entre os domicílios com insegurança alimentar e insegurança alimentar grave na região Norte, diferente da Centro-Oeste onde o consumo dessa mesma hortaliça era maior em domicílios seguros. A bertalha, na região Sudeste, só foi consumida entre adolescentes em situação de segurança alimentar. Dentre as hortaliças analisadas, apenas a taioba não apresentou diferença de consumo segundo situação de segurança alimentar (Tabela 2). Em relação às frutas nacionais, a uva foi a mais consumida por adolescentes seguros e o coco, pelos inseguros (Tabela 3).

Na região Norte a fruta mais consumida foi o cupuaçu, principalmente na situação de insegurança alimentar. $\mathrm{O}$ mesmo foi observado com as outras frutas da região, exceto para a siriguela, mais consumida pelos adolescentes em segurança alimentar. Nas regiões Nordeste, Centro-Oeste e Sudeste as frutas seguiram a mesma tendência de consumo observada entre as

Tabela 1. Situação de insegurança alimentar (\%) segundo as características socioeconômicas dos adolescentes nas capitais brasileiras e Distrito Federal, 2013.

\begin{tabular}{|c|c|c|c|c|c|c|c|}
\hline Características & $n$ & $\%^{*}$ & SA $(\%)^{*}$ & $\mathrm{IAL}(\%)^{*}$ & IAM $(\%)^{*}$ & IAG $(\%)^{*}$ & $p$ \\
\hline \multicolumn{8}{|l|}{ Sexo } \\
\hline Feminino & 7822 & 55,7 & 77,1 & 16,6 & 3,9 & 2,3 & \multirow{2}{*}{$<0,001$} \\
\hline Masculino & 6868 & 44,3 & 73,6 & 18,5 & 5,1 & 2,9 & \\
\hline \multicolumn{8}{|l|}{ Tipo de escola } \\
\hline Pública & 10684 & 78,2 & 71,5 & 20,3 & 5,3 & 3,0 & \multirow{2}{*}{$<0,001$} \\
\hline Privada & 4006 & 21,8 & 86,1 & 10,0 & 2,3 & 1,5 & \\
\hline \multicolumn{8}{|l|}{ Escolaridade materna } \\
\hline Analfabeta & 388 & 3,1 & 54,9 & 22,4 & 8,8 & 13,9 & \multirow{4}{*}{$<0,001$} \\
\hline Ensino Fundamental & 2814 & 27,8 & 67,3 & 22,9 & 6,1 & 3,7 & \\
\hline Ensino Médio & 3994 & 32,0 & 75,6 & 18,0 & 4,6 & 1,9 & \\
\hline Ensino Superior & 5123 & 37,1 & 82,6 & 13,1 & 2,7 & 1,6 & \\
\hline \multicolumn{8}{|l|}{ Região geográfica } \\
\hline Norte & 4381 & 10,9 & 68,2 & 21,9 & 6,1 & 3,9 & \multirow{5}{*}{$<0,001$} \\
\hline Nordeste & 4440 & 21,5 & 76,4 & 16,8 & 4,5 & 2,2 & \\
\hline Centro-Oeste & 2302 & 12,9 & 77,9 & 16,6 & 3,5 & 2,1 & \\
\hline Sudeste & 2287 & 49,0 & 82,3 & 13,0 & 3,1 & 1,5 & \\
\hline Sul & 1280 & 5,8 & 80,5 & 14,3 & 3,0 & 2,2 & \\
\hline
\end{tabular}

Nota: *Percentuais com expansão da amostra.

SA: Segurança Alimentar; IAL: Insegurança Alimentar Leve; IAM: Insegurança Alimentar Moderada; IAG: Insegurança Alimentar Grave. 
Tabela 2. Prevalência de consumo de hortaliças regionais e situação de insegurança alimentar domiciliar dos adolescentes nas capitais brasileiras e Distrito Federal, 2013.

\begin{tabular}{|c|c|c|c|c|c|c|c|c|}
\hline \multirow{2}{*}{ Região } & \multirow{2}{*}{ Hortaliças } & \multirow{2}{*}{$\mathrm{N}$} & \multicolumn{2}{|c|}{ Consome } & \multirow{2}{*}{$\frac{\mathrm{SA}}{\%}$} & \multirow{2}{*}{$\frac{\mathrm{IA}}{\%}$} & & \multirow{2}{*}{$\begin{array}{c}\text { IAG/SA } \\
\operatorname{RP}(I C 95 \%)\end{array}$} \\
\hline & & & $n$ & $\%^{*}$ & & & & \\
\hline \multirow[t]{6}{*}{ Nacionais } & Mandioca & 13708 & 11805 & 83,1 & 83,0 & 83,4 & 1,041 & $(1,038-1,043)$ \\
\hline & Batata-doce & 13467 & 8644 & 62,2 & 60,4 & 68,3 & 1,321 & $(1,317-1,325)$ \\
\hline & Couve & 10407 & 6265 & 61,4 & 61,3 & 62,1 & 1,108 & $(1,104-1,113)$ \\
\hline & Cará & 4637 & 2596 & 55,5 & 54,9 & 57,4 & 1,052 & $(1,045-1,060)$ \\
\hline & Quiabo & 12343 & 4665 & 38,8 & 37,7 & 42,7 & 1,224 & $(1,216-1,231)$ \\
\hline & Jiló & 8738 & 1205 & 14,5 & 13,8 & 17,1 & 1,245 & $(1,227-1,264)$ \\
\hline \multirow[t]{6}{*}{ Norte } & Jambu & 803 & 602 & 79,2 & 79,6 & 78,6 & 1,044 & $(1,035-1,053)$ \\
\hline & Chicória & 2982 & 1832 & 59,6 & 57,9 & 62,8 & 1,349 & $(1,339-1,358)$ \\
\hline & Serralha & 131 & 78 & 53,9 & 57,3 & 44,7 & 0,401 & $(0,328-0,490)$ \\
\hline & Alfavaca & 1524 & 659 & 43,6 & 41,8 & 46,8 & 1,469 & $(1,449-1,489)$ \\
\hline & Caruru & 334 & 289 & 42,1 & 37,8 & 50,3 & 1,764 & $(1,725-1,805)$ \\
\hline & Gueroba & 239 & 74 & 27,3 & 22,8 & 41,9 & 2,500 & $(2,320-2,695)$ \\
\hline \multirow[t]{5}{*}{ Nordeste } & Vinagreira & 462 & 357 & 78,7 & 77,2 & 82,4 & 1,211 & $(1,202-1,221)$ \\
\hline & Inhame & 3175 & 2324 & 72,6 & 72,2 & 73,7 & 1,018 & $(1,010-1,026)$ \\
\hline & João-Gomes & 180 & 94 & 58,0 & 57,1 & 60,4 & 0,464 & $(0,439-0,491)$ \\
\hline & Jerimum/Abóbora & 3529 & 1845 & 52,8 & 51,1 & 57,9 & 1,260 & $(1,248-1,272)$ \\
\hline & Maxixe & 3411 & 895 & 25,2 & 24,4 & 27,8 & 1,279 & $(1,254-1,303)$ \\
\hline \multirow[t]{6}{*}{ Centro-Oeste } & Abóbora/Jerimum & 1141 & 675 & 59,7 & 57,4 & 65,6 & 1,509 & $(1,496-1,522)$ \\
\hline & Inhame & 458 & 240 & 49,4 & 48,4 & 51,5 & 1,454 & $(1415-1,495)$ \\
\hline & Serralha & 95 & 39 & 44,0 & 43,1 & 47,6 & 0,808 & $(0,776-0,840)$ \\
\hline & Maxixe & 741 & 287 & 40,4 & 36,5 & 49,3 & 1,121 & $(1,073-1,172)$ \\
\hline & Taioba & 217 & 71 & 37,9 & 37,4 & 40,1 & 1,712 & $(1,665-1,760)$ \\
\hline & Gueroba & 829 & 236 & 31,7 & 31,1 & 34,0 & 0,990 & $(0,962-1,018)$ \\
\hline \multirow[t]{6}{*}{ Sudeste } & Batata baroa & 317 & 207 & 69,5 & 68,3 & 75,7 & 1,424 & $(1,416-1,433)$ \\
\hline & Beldroega & 35 & 12 & 38,9 & 29,4 & 100,0 & 3,400 & $(3,347-3,454)$ \\
\hline & Bertalha & 48 & 24 & 34,0 & 40,2 & 0,0 & & - \\
\hline & Mostarda & 1347 & 523 & 29,1 & 29,6 & 26,9 & 2,023 & $(2,002-2,045)$ \\
\hline & Rúcula & 701 & 377 & 50,2 & 49,8 & 51,9 & 1,274 & $(1,261-1,287)$ \\
\hline & Taioba** & 722 & 304 & 43,3 & 43,4 & 43,1 & 0,410 & $(0,378-0,444)$ \\
\hline \multirow[t]{3}{*}{ Sul } & Repolho & 1219 & 889 & 76,0 & 75,0 & 79,9 & 1,216 & $(1,208-1,224)$ \\
\hline & Almeirão-Roxo & 226 & 93 & 43,1 & 37,9 & 59,7 & 1,741 & $(1,703-1,780)$ \\
\hline & Raditi & 416 & 166 & 39,6 & 37,3 & 48,9 & 1,588 & $(1,550-1,628)$ \\
\hline
\end{tabular}

Nota: *Valor com expansão da amostra; **Valor de $p$ não significativo.

SA: Segurança Alimentar; IA: Insegurança Alimentar (leve, moderada ou grave); RP: Razão de Prevalência; IC95\%: Intervalo de Confiança de 95\%.

hortaliças, ou seja, o mesmo era maior entre adolescentes em situação de insegurança alimentar e insegurança alimentar grave. Foram exceções a pitanga, na região Nordeste, e a guabiroba, na Centro-Oeste, mais consumidas pelos seguros. No Sudeste, a diferença de consumo entre seguros e inseguros não foi significativa para o caqui (Tabela 3).

No Sul, ao contrário do observado nas demais regiões, os adolescentes seguros conso- mem mais frutas regionais quando comparados àqueles em insegurança alimentar e insegurança alimentar grave. As frutas com maior ingestão entre os adolescentes inseguros graves foram apenas a nectarina e a jabuticaba (Tabela 3).

Quando analisadas as preparações nacionais foi observado alto consumo pelos adolescentes, exceto a dobradinha, pouco consumida em relação às demais (49,6\%). O consumo dessas preparações seguiu o padrão das frutas e 
Tabela 3. Consumo de frutas regionais e situação de insegurança alimentar domiciliar dos adolescentes nas capitais brasileiras e Distrito Federal, 2013.

1 de 2

\begin{tabular}{|c|c|c|c|c|c|c|c|c|}
\hline \multirow{2}{*}{ Região } & \multirow{2}{*}{ Frutas } & \multirow{2}{*}{$\mathrm{N}$} & \multicolumn{2}{|c|}{ Consome } & \multirow{2}{*}{$\begin{array}{l}\text { SA } \\
\%\end{array}$} & \multirow{2}{*}{$\frac{\mathrm{IA}}{\%}$} & & \multirow{2}{*}{$\begin{array}{c}I A G / S A \\
\operatorname{RP}(I C 95 \%)\end{array}$} \\
\hline & & & $\mathrm{n}$ & $\%^{*}$ & & & & \\
\hline \multirow[t]{2}{*}{ Nacionais } & Uva & 14427 & 13800 & 95,6 & 95,8 & 95,5 & 1,009 & $(1,008-1,010)$ \\
\hline & Coco & 14320 & 13019 & 88,4 & 88,9 & 90,8 & 1,056 & $(1,055-1,058)$ \\
\hline \multirow[t]{13}{*}{ Norte } & Cupuaçu & 3663 & 3376 & 93,0 & 92,5 & 93,9 & 1,030 & $(1,028-1,033)$ \\
\hline & Banana pacovã & 2113 & 1887 & 90,8 & 90,2 & 92,0 & 1,094 & $(1,092-1,096)$ \\
\hline & Açaí & 3648 & 3124 & 87,8 & 86,8 & 89,7 & 1,048 & $(1,045-1,052)$ \\
\hline & Jambo & 941 & 830 & 86,3 & 85,1 & 88,3 & 1,124 & $(1,119-1,128)$ \\
\hline & Mangaba & 238 & 178 & 79,6 & 74,5 & 90,1 & 1,119 & $(1,077-1,164)$ \\
\hline & Tucumã & 1512 & 1042 & 77,8 & 76,4 & 80,4 & 1,079 & $(1,071-1,086)$ \\
\hline & Pupunha & 1652 & 1127 & 72,1 & 70,8 & 74,5 & 1,156 & $(1,147-1,164)$ \\
\hline & Buriti & 504 & 351 & 69,8 & 67,6 & 76,6 & 1,140 & $(1,115-1,166)$ \\
\hline & Bacuri & 668 & 400 & 63,0 & 62,5 & 64,0 & 1,083 & $(1,068-1,098)$ \\
\hline & Murici & 1182 & 732 & 62,7 & 61,3 & 65,4 & 1,189 & $(1,176-1,202)$ \\
\hline & Biriba & 550 & 339 & 60,7 & 58,6 & 64,4 & 1,108 & $(1,091-1,125)$ \\
\hline & Siriguela & 277 & 153 & 56,0 & 53,9 & 62,6 & 0,792 & $(0,710-0,883)$ \\
\hline & Taperebá ou cajá & 2543 & 1533 & 56,0 & 54,2 & 59,4 & 1,507 & $(1,495-1,518)$ \\
\hline \multirow[t]{15}{*}{ Nordeste } & Manga & 1051 & 945 & 89,9 & 89,4 & 91,5 & 1,119 & $(1,117-1,120)$ \\
\hline & Jambo & 503 & 440 & 89,6 & 88,2 & 93,2 & 1,133 & $(1,131-1,136)$ \\
\hline & Siriguela & 4013 & 3279 & 82,8 & 81,6 & 86,3 & 1,087 & $(1,083-1,092)$ \\
\hline & Pitomba & 1300 & 1011 & 77,2 & 75,5 & 82,6 & 1,110 & $(1,098-1,123)$ \\
\hline & Pinha & 975 & 715 & 74,0 & 71,5 & 82,6 & 1,312 & $(1,303-1,320)$ \\
\hline & Umbu & 1714 & 1159 & 72,8 & 71,5 & 77,5 & 1,255 & $(1,247-1,263)$ \\
\hline & Cajá & 3451 & 2464 & 70,4 & 69,9 & 72,0 & 1,035 & $(1,028-1,043)$ \\
\hline & Murici & 450 & 281 & 68,7 & 64,8 & 79,1 & 0,978 & $(0,957-1,000)$ \\
\hline & Caju & 910 & 619 & 67,4 & 69,2 & 62,2 & 0,950 & $(0,936-0,964)$ \\
\hline & Graviola & 2736 & 1761 & 66,4 & 64,8 & 71,2 & 1,013 & $(1,002-1,024)$ \\
\hline & Buriti & 443 & 226 & 56,6 & 51,8 & 69,0 & 1,588 & $(1,566-1,610)$ \\
\hline & Jenipapo & 349 & 188 & 52,9 & 52,7 & 54,3 & 0,994 & $(0,969-1,020)$ \\
\hline & Mangaba & 1808 & 937 & 51,5 & 52,1 & 49,9 & 1,041 & $(1,024-1,058)$ \\
\hline & Fruta-Pão & 474 & 163 & 41,1 & 40,0 & 44,5 & 0,889 & $(0,854-0,926)$ \\
\hline & Pitanga & 507 & 189 & 36,5 & 38,7 & 28,3 & 0,460 & $(0,435-0,487)$ \\
\hline \multirow[t]{9}{*}{ Centro-Oeste } & Tangerina/Mexerica & 1044 & 994 & 96,1 & 95,3 & 98,1 & 1,049 & $(1,048-1,050)$ \\
\hline & Manga & 2258 & 2068 & 93,5 & 93,2 & 94,7 & 0,997 & $(0,993-1,001)$ \\
\hline & Abacate & 1043 & 805 & 80,1 & 78,3 & 84,7 & 1,119 & $(1,108-1,130)$ \\
\hline & Araticum/Articum & 764 & 541 & 73,1 & 70,8 & 79,6 & 1,047 & $(1,036-1,057)$ \\
\hline & Jaca & 1185 & 716 & 63,3 & 60,5 & 70,7 & 0,898 & $(0,882-0,916)$ \\
\hline & Pequi & 2724 & 1696 & 61,0 & 58,7 & 68,1 & 1,427 & $(1,418-1,437)$ \\
\hline & Cagaita & 430 & 214 & 57,1 & 53,3 & 70,9 & 0,925 & $(0,900-0,952)$ \\
\hline & Guabiroba & 554 & 150 & 30,2 & 31,1 & 26,2 & 0,531 & $(0,505-0,558)$ \\
\hline & Jenipapo & 661 & 112 & 19,0 & 17,5 & 25,2 & 1,153 & $(1,100-1,208)$ \\
\hline \multirow[t]{11}{*}{ Sudeste } & Tangerina/Mexerica & 968 & 914 & 96,9 & 96,9 & 97,0 & 0,929 & $(0,921-0,937)$ \\
\hline & Abacaxi & 162 & 147 & 93,5 & 93,8 & 91,6 & 0,533 & $(0,461-0,616)$ \\
\hline & Manga & 1427 & 1283 & 87,9 & 87,3 & 90,2 & 1,106 & $(1,103-1,109)$ \\
\hline & Morango & 161 & 143 & 86,4 & 88,7 & 75,0 & 1,127 & $(1,122-1,133)$ \\
\hline & Abacate & 2171 & 1436 & 68,6 & 67,8 & 72,0 & 1,129 & $(1,123-1,135)$ \\
\hline & Mamão & 157 & 104 & 67,4 & 72,3 & 43,2 & 1,383 & $(1,371-1,394)$ \\
\hline & Jabuticaba & 2011 & 1461 & 66,8 & 66,1 & 69,3 & 0,707 & $(0,699-0,714)$ \\
\hline & Caqui $^{* *}$ & 2014 & 1239 & 65,7 & 65,7 & 65,7 & 1,159 & $(1,153-1,166)$ \\
\hline & Caju & 785 & 411 & 53,5 & 51,6 & 61,9 & 0,617 & $(0,606-0,628)$ \\
\hline & Jaca & 1155 & 480 & 45,5 & 42,6 & 58,5 & 1,038 & $(1,027-1,050)$ \\
\hline & Abiu & 142 & 66 & 43,1 & 45,6 & 36,4 & 1,665 & $(1,646-1,684)$ \\
\hline
\end{tabular}


Tabela 3. Consumo de frutas regionais e situação de insegurança alimentar domiciliar dos adolescentes nas capitais brasileiras e Distrito Federal, 2013.

2 de 2

\begin{tabular}{|c|c|c|c|c|c|c|c|c|}
\hline \multirow{2}{*}{ Região } & \multirow{2}{*}{ Frutas } & \multirow{2}{*}{$\mathrm{N}$} & \multicolumn{2}{|c|}{ Consome } & \multirow{2}{*}{$\begin{array}{l}\text { SA } \\
\%\end{array}$} & \multirow{2}{*}{$\begin{array}{l}\mathrm{A} \\
\%\end{array}$} & & \multirow{2}{*}{$\begin{array}{c}\text { IAG/SA } \\
\operatorname{RP}(I C 95 \%)\end{array}$} \\
\hline & & & $n$ & $\%^{*}$ & & & & \\
\hline \multirow[t]{7}{*}{ Sul } & Tangerina/Mexerica & 1240 & 1142 & 92,4 & 92,9 & 90,5 & 0,847 & $(0,838-0,855)$ \\
\hline & Pinhão & 520 & 465 & 90,3 & 90,8 & 88,3 & 0,729 & $(0,714-0,744)$ \\
\hline & Pêssego & 1229 & 980 & 80,1 & 79,2 & 83,9 & 0,890 & $(0,878-0,901)$ \\
\hline & Amora & 1210 & 912 & 76,8 & 77,0 & 76,0 & 0,903 & $(0,892-0,915)$ \\
\hline & Ameixa & 1188 & 857 & 72,3 & 72,1 & 73,3 & 0,865 & $(0,851-0,879)$ \\
\hline & Nectarina & 796 & 547 & 70,5 & 69,2 & 75,7 & 1,153 & $(1,139-1,166)$ \\
\hline & Jabuticaba & 627 & 312 & 43,8 & 43,7 & 44,6 & 1,186 & $(1,146-1,228)$ \\
\hline
\end{tabular}

Nota: *Valor com expansão da amostra; ** Valor de $p$ não significativo.

SA: Segurança Alimentar; IA: Insegurança Alimentar (leve, moderada ou grave); RP: Razão de Prevalência; IC95\%: Intervalo de Confiança de $95 \%$.

hortaliças regionais, sendo maior entre os adolescentes inseguros (Tabela 4).

Na região Norte, a preparação mais consumida foi a tapioca. Nessa região, assim como na Nordeste, as preparações regionais foram, em sua maioria, mais consumidas por adolescentes em situação de segurança alimentar. O pão de queijo foi a preparação mais consumida na região Centro-Oeste, principalmente entre os adolescentes inseguros. No Sudeste essa preparação também foi a mais consumida, porém pelos adolescentes seguros. No Sul, o churrasco apresentou maior prevalência de consumo, com o mesmo percentual entre seguros e inseguros. No entanto, quando comparados inseguros graves com seguros, os primeiros a consumiam menos ( $R P=0,95)$.

\section{I S C U S S Ã O}

Este estudo teve como público os adolescentes brasileiros e a amostra foi composta em sua maioria por indivíduos do sexo feminino, de escolas públicas e com média de idade de 14 anos, aproximadamente, características similares à Pesquisa Nacional de Saúde do Escolar (PeNSE) $2012^{13}$. Em relação à escolaridade materna, proxy de renda, a presente pesquisa apontou um percentual elevado de mães com ensino superior $(29,4 \%)$, maior que o encontrado na PeNSE, onde somente $8,9 \%$ delas tinham esse grau de instru- ção, bem como no Censo $^{14}$, onde $12,5 \%$ das mulheres acima de 25 anos afirmaram possuir nível superior completo ${ }^{13,14}$. Uma das possíveis explicações para essas diferenças é que a amostra, diferentemente do Censo e da PeNSE, compreendeu apenas as capitais brasileiras e o Distrito Federal, o que pode ter elevado o nível de escolaridade, uma vez que as populações destas cidades apresentam características diferentes das do Brasil em geral ${ }^{14}$.

A insegurança alimentar foi mais prevalente nos domicílios com alunos que estudavam em escolas públicas e que tinham mães com baixa escolaridade. Esta última característica é considerada um importante fator de proteção para a saúde de crianças e adolescentes, como proxy das

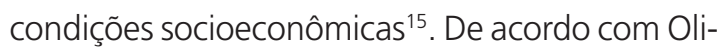
veira et al. ${ }^{16}$, os pais dos alunos de escolas privadas possuem um grau de instrução maior e, por consequência, maior renda; este fato justifica uma menor prevalência de insegurança alimentar entre esses alunos. A insegurança alimentar é mais presente em famílias com piores condições de vida (renda e escolaridade), como observado na Pesquisa Nacional por Amostra de Domicílios $(P N A D)^{17}$. A região com maior prevalência de insegurança alimentar foi a Norte, o que condiz com o fato de esta ser uma das regiões com menor renda per capita no Brasil14,17.

O consumo de hortaliças e frutas regionais, em geral, foi maior entre adolescentes residentes 
Tabela 4. Consumo de preparações regionais e situação de insegurança alimentar domiciliar dos adolescentes nas capitais brasileiras e Distrito Federal, 2013.

1 de 2

\begin{tabular}{|c|c|c|c|c|c|c|c|c|}
\hline \multirow{2}{*}{ Região } & \multirow{2}{*}{ Preparações } & \multirow{2}{*}{$\mathrm{N}$} & \multicolumn{2}{|c|}{ Consome } & \multirow{2}{*}{$\begin{array}{l}\text { SA } \\
\%\end{array}$} & \multirow{2}{*}{$\frac{\mathrm{IA}}{\%}$} & & \multirow{2}{*}{$\begin{array}{c}\text { IAG/SA } \\
\operatorname{RP}(I C 95 \%)\end{array}$} \\
\hline & & & $\mathrm{n}$ & $\%^{*}$ & & & & \\
\hline \multirow[t]{5}{*}{ Nacional } & Feijoada & 14146 & 12084 & 83,6 & 83,0 & 85,6 & 1,041 & $(1,039-1,044)$ \\
\hline & Maria Izabel ou arroz carreteiro & 10500 & 8829 & 80,8 & 80,1 & 83,4 & 1,102 & $(1,100-1,105)$ \\
\hline & Mungunzá ou canjica & 12723 & 9567 & 74,8 & 72,8 & 82,3 & 1,140 & $(1,137-1,143)$ \\
\hline & Cozido & 11998 & 9045 & 74,0 & 71,9 & 80,8 & 1,084 & $(1,080-1,087)$ \\
\hline & Dobradinha & 8595 & 4237 & 49,6 & 47,7 & 55,8 & 1,269 & $(1,262-1,275)$ \\
\hline \multirow[t]{10}{*}{ Norte } & Tapioca & 3655 & 3520 & 96,7 & 96,6 & 96,8 & 1,001 & $(0,999-1,004)$ \\
\hline & Feijão tropeiro & 615 & 581 & 94,3 & 94,9 & 92,3 & 0,890 & $(0,876-0,903)$ \\
\hline & Carne de sol & 4192 & 3882 & 93,1 & 93,5 & 92,2 & 0,933 & $(0,930-0,937)$ \\
\hline & Pamonha & 604 & 533 & 90,8 & 92,1 & 86,5 & 0,635 & $(0,615-0,656)$ \\
\hline & Baião de dois & 3470 & 3120 & 89,4 & 89,5 & 89,3 & 1,010 & $(1,006-1,013)$ \\
\hline & Vaca atolada & 966 & 803 & 82,3 & 83,6 & 78,6 & 0,836 & $(0,818-0,854)$ \\
\hline & Vatapá & 3565 & 2896 & 82,2 & 81,3 & 83,8 & 1,118 & $(1,114-1,123)$ \\
\hline & Caldeirada de peixe & 3275 & 2542 & 77,5 & 75,4 & 81,6 & 1,116 & $(1,111-1,122)$ \\
\hline & Frango no tucupi & 775 & 555 & 75,0 & 74,5 & 76,0 & 1,209 & $(1,200-1,217)$ \\
\hline & Curau & 457 & 342 & 74,2 & 74,8 & 71,8 & 0,667 & $(0,630-0,705)$ \\
\hline \multirow[t]{10}{*}{ Nordeste } & Carne de sol & 3956 & 3826 & 96,2 & 96,3 & 95,8 & 1,010 & $(1,008-1,012)$ \\
\hline & Cuscuz & 3417 & 3215 & 93,1 & 92,2 & 95,8 & 0,996 & $(0,993-1,000)$ \\
\hline & Baião de dois & 1412 & 1299 & 91,9 & 91,5 & 93,3 & 0,996 & $(0,991-1,001)$ \\
\hline & Escondidinho de carne seca & 2287 & 1872 & 91,5 & 92,7 & 87,0 & 0,918 & $(0,904-0,933)$ \\
\hline & Arrumadinho** & 398 & 357 & 91,4 & 91,4 & 91,3 & 1,018 & $(1,010-1,025)$ \\
\hline & Tapioca & 4291 & 3010 & 91,2 & 91,2 & 91,1 & 0,970 & $(0,967-0,974)$ \\
\hline & Acarajé & 391 & 340 & 88,1 & 86,7 & 92,1 & 1,068 & $(1,061-1,076)$ \\
\hline & Feijão verde & 1328 & 1170 & 87,7 & 88,1 & 86,5 & 0,943 & $(0,933-0,954)$ \\
\hline & Pirão & 3712 & 3058 & 83,4 & 82,8 & 85,2 & 0,995 & $(0,990-1,000)$ \\
\hline & Paçoca & 2120 & 1739 & 82,7 & 80,5 & 89,5 & 1,104 & $(1,096-1,112)$ \\
\hline \multirow[t]{10}{*}{ Centro-Oeste } & Pão de queijo & 2269 & 2225 & 98,6 & 98,5 & 98,7 & 0,980 & $(0,977-0,982)$ \\
\hline & Feijão tropeiro & 1997 & 1787 & 92,4 & 92,5 & 92,0 & 0,988 & $(0,984-0,992)$ \\
\hline & Mané pelado ou bolo de mandioca & 171 & 144 & 90,1 & 87,7 & 97,7 & 1,140 & $(1,138-1,142)$ \\
\hline & Pamonha & 2221 & 1902 & 89,3 & 88,9 & 90,5 & 1,113 & $(1,111-1,115)$ \\
\hline & Empadão goiano & 890 & 779 & 86,8 & 86,7 & 87,3 & 1,062 & $(1,055-1,069)$ \\
\hline & Galinhada & 179 & 161 & 85,1 & 94,3 & 97,4 & 1,061 & $(1,060-1,062)$ \\
\hline & Frango com açafrão & 1054 & 863 & 83,9 & 82,6 & 87,9 & 1,049 & $(1,042-1,056)$ \\
\hline & Vaca atolada & 1929 & 1629 & 79,0 & 76,9 & 85,2 & 0,960 & $(0,952-0,968)$ \\
\hline & Baião de dois & 165 & 131 & 77,1 & 75,7 & 80,9 & 1,321 & $(1,318-1,325)$ \\
\hline & Canjica & 1964 & 1478 & 76,8 & 74,2 & 85,5 & 1,218 & $(1,212-1,223)$ \\
\hline \multirow[t]{10}{*}{ Sudeste } & Pão de queijo & 2246 & 2163 & 95,7 & 96,4 & 93,1 & 0,964 & $(0,962-0,967)$ \\
\hline & Picadinho de carne & 1253 & 1107 & 88,2 & 87,1 & 92,8 & 1,012 & $(1,007-1,016)$ \\
\hline & Peixe a milanesa & 572 & 509 & 85,9 & 85,3 & 88,2 & 0,982 & $(0,975-0,989)$ \\
\hline & Moqueca capixaba & 154 & 126 & 84,0 & 82,5 & 91,6 & 1,212 & $(1,204-1,220)$ \\
\hline & Torta capixaba & 147 & 110 & 79,3 & 78,0 & 86,3 & & \\
\hline & Frango com quiabo & 785 & 616 & 75,8 & 74,2 & 83,4 & 0,637 & $(0,620-0,655)$ \\
\hline & Feijão tropeiro & 1347 & 1105 & 75,6 & 74,6 & 80,1 & 0,986 & $(0,979-0,994)$ \\
\hline & Angu $^{* *}$ & 788 & 576 & 75,0 & 75,1 & 74,9 & 0,791 & $(0,775-0,808)$ \\
\hline & Bolo de mandioca ou mané pelado & 1629 & 1114 & 74,1 & 73,2 & 77,4 & 1,116 & $(1,111-1,122)$ \\
\hline & Peixe cozido & 400 & 274 & 69,6 & 71,1 & 63,6 & 0,693 & $(0,680-0,706)$ \\
\hline
\end{tabular}


Tabela 4. Consumo de preparações regionais e situação de insegurança alimentar domiciliar dos adolescentes nas capitais brasileiras e Distrito Federal, 2013.

\begin{tabular}{|c|c|c|c|c|c|c|c|c|}
\hline \multirow{2}{*}{ Região } & \multirow{2}{*}{ Preparações } & \multirow{2}{*}{$\mathrm{N}$} & \multicolumn{2}{|c|}{ Consome } & \multirow{2}{*}{$\begin{array}{l}\text { SA } \\
\%\end{array}$} & \multirow{2}{*}{$\frac{\mathrm{IA}}{\%}$} & & \multirow{2}{*}{$\begin{array}{c}\text { IAG/SA } \\
\operatorname{RP}(I C 95 \%)\end{array}$} \\
\hline & & & $\mathrm{n}$ & $\%^{*}$ & & & & \\
\hline \multirow[t]{10}{*}{ Sul } & Churrasco** & 1257 & 1229 & 98,2 & 98,2 & 98,2 & 0,959 & $(0,954-0,963)$ \\
\hline & Polenta** & 527 & 464 & 89,0 & 89,0 & 88,9 & 1,124 & $(1,122-1,126)$ \\
\hline & Cuca & 328 & 266 & 82,3 & 82,8 & 80,2 & 0,935 & $(0,916-0,954)$ \\
\hline & Feijão campeiro & 418 & 331 & 78,0 & 77,2 & 81,2 & 0,948 & $(0,935-0,962)$ \\
\hline & Virado de feijão** & 356 & 263 & 72,6 & 72,5 & 73,0 & 1,379 & $(1,373-1,385)$ \\
\hline & Matambre & 154 & 105 & 70,8 & 74,5 & 55,9 & 1,102 & $(1,079-1,126)$ \\
\hline & Sagu & 1203 & 834 & 69,8 & 69,9 & 69,3 & 1,131 & $(1,118-1,145)$ \\
\hline & Quirrera lapiana & 217 & 148 & 68,2 & 65,9 & 75,3 & 1,518 & $(1,511-1,525)$ \\
\hline & Peixe na telha & 472 & 329 & 65,5 & 66,3 & 62,3 & 1,023 & $(1,003-1,043)$ \\
\hline & Ostra gratinada** & 291 & 193 & 65,1 & 64,9 & 65,8 & 0,635 & $(0,589-0,685)$ \\
\hline
\end{tabular}

Nota: "Valor com expansão da amostra; ${ }^{* *}$ Valor de $p$ não significativo.

SA: Segurança Alimentar; IA: Insegurança Alimentar (leve, moderada ou grave); RP: Razão de Prevalência; IC95\%: Intervalo de Confiança de $95 \%$.

em domicílios com insegurança alimentar, se comparados aos seguros, em todas as regiões geográficas. Tal fato é diferente do observado por Martins $^{18}$, que investigou o consumo de alimentos regionais e situação de segurança alimentar no interior do Ceará, observando um decréscimo na utilização desses alimentos (essencialmente frutas, hortaliças e tubérculos) à medida que a aumentava a gravidade da insegurança alimentar. Cabe ressaltar que as amostras dos estudos são diferentes, um foi realizado em município do interior do Nordeste, região semiárida com altas prevalências de insegurança alimentar, e o outro compreendeu o território nacional, com amostra apenas nas capitais dos estados e Distrito Federal, fato que limita a comparação dos dois resultados.

Apesar de a insegurança alimentar comprometer o acesso a uma alimentação em termos qualiquantitativos, isso parece não ser verdadeiro para a alimentação regional, em especial frutas e hortaliças. Vários fatores podem estar influenciando o maior consumo desses alimentos na situação de insegurança: disponibilidade gratuita nas proximidades do domicílio, sazonalidade, acesso a estes por meio de banco de alimentos e maior proteção em relação ao consumo de alimentos industrializados devido à restrição econômica.
Os alimentos regionais têm como principais características o fácil acesso, o baixo custo e alto valor nutritivo e seu consumo deve ser estimulado e valorizado ${ }^{19}$. Eles podem, muitas vezes, estar disponíveis nas residências através da extração ou do plantio, não sendo necessária a sua compra. Outro fator importante que poderia facilitar o maior consumo na situação de insegurança alimentar é o baixo custo, sendo uma boa alternativa para famílias de baixa renda, uma vez que a restrição de consumo de frutas e hortaliças em famílias menos favorecidas normalmente está ligado à impossibilidade financeira de compra ${ }^{20}$. Os bancos de alimentos são uma outra forma de acesso a esses alimentos, um equipamento público que tem como função receber, selecionar, processar, quando necessário, e distribuir os alimentos arrecadados à população, seja através do fornecimento de refeições prontas ou do repasse direto às famílias em condições de pobreza ${ }^{21}$.

Outra política pública que pode contribuir para a inserção dos alimentos regionais nos hábitos alimentares dos estudantes é o Programa Nacional de Alimentação Escolar (PNAE), que tem como um de seus princípios a preservação de práticas tradicionais, da cultura e da preferência alimentar local. Esse programa tem uma grande cobertura no País e utiliza o ambiente escolar para 
promoção da alimentação saudável ${ }^{22}$. Assim, como a maior parte dos estudantes participantes da pesquisa é de escolas públicas, o programa pode estar contribuindo para um maior consumo de frutas e hortaliças regionais e para a diversidade da dieta, bem como para a preservação da cultura alimentar regional entre os adolescentes ${ }^{23}$.

Deve-se considerar, ainda, que a insegurança alimentar pode ser fator de proteção em relação ao consumo de alimentos industrializados devido a restrições financeiras. Estudos mostram que os hábitos alimentares dos adolescentes estão cada vez menos saudáveis, principalmente entre aqueles que pertencem às classes econômicas mais favorecidas, devido ao maior acesso aos alimentos industrializados ${ }^{3,4,24}$. Sabe-se que o hábito alimentar dessa população é caracterizado pela ingestão de alimentos ricos em gorduras, açúcares e sódio, contando apenas com uma pequena participação de frutas e hortaliças ${ }^{25}$. Porém entre os jovens provenientes de famílias menos favorecidas, o consumo de alimentos marcadores de dieta saudável, como o arroz e o feijão, é mais frequente, embora a ingestão de frutas e hortaliças seja menos habitual26,27.

Em relação às preparações nacionais, o consumo seguiu o mesmo padrão das frutas e hortaliças, sendo mais consumidas entre adolescentes em insegurança alimentar. Tratam-se de preparações tradicionais que refletem a identidade brasileira, como a feijoada e o arroz carreteiro, e são passadas de geração em geração como hábito alimentar. São, normalmente, de baixo custo e amplamente conhecidas ${ }^{28}$. A dobradinha ou buchada foi a preparação menos consumida pelos adolescentes neste estudo e sua forma de preparo, sabor e odor peculiar podem ter contribuído para isso, uma vez que os mesmos são fatores determinantes na escolha alimentar ${ }^{29,30}$.

Já em relação às preparações regionais, o comportamento foi diferente, sendo mais consumidas por adolescentes em domicílios classificados como seguros. Grande parte das preparações apresentadas eram pratos mais elaborados, muitos com carnes em sua composição, o que pode limitar o acesso pelos adolescentes em insegurança alimentar devido ao maior custo. Um exemplo disso é o churrasco no Sul, preparação mais consumida entre adolescentes seguros quando comparados aos com insegurança alimentar grave. Importante salientar, também, que, de acordo com a Pesquisa de Orçamentos Familiares $^{2}$-, a participação da carne bovina no total de calorias consumidas cresce à medida que aumenta o rendimento familiar.

Assim, ressalta-se a importância da promoção do consumo de alimentos produzidos localmente como referência cultural da comunidade, em especial frutas e hortaliças regionais, levando em consideração o respeito à diversidade cultural que compõe o amplo conceito de segurança alimentar e nutricional. Logo, ações que promovam o cultivo e o consumo de alimentos regionais e saudáveis, seja por meio da agricultura familiar, uso de modelos agroecológicos ou diversificação da dieta, contribuem para o resgate do valor nutricional e cultural desses alimentos, preservando a biodiversidade de cada região e promovendo a sustentabilidade ${ }^{5}$.

\section{O N CLUS Ã O}

Este estudo apresentou a insegurança alimentar associada ao maior consumo de alimentos marcadores de dieta saudável, como frutas e hortaliças regionais. No entanto há limitações que devem ser levadas em consideração. A pesquisa apenas verificou presença desses alimentos na dieta dos adolescentes e não a quantidade e frequência de consumo, informações estas que poderiam desenhar um panorama do consumo usual do individuo com maior acurácia. Também analisou um número limitado de frutas e hortaliças regionais, o que pode subestimar o consumo deste tipo de alimento. Outra limitação é o possível viés de conveniência social, onde os adolescentes podem ter informado o consumo socialmente aceito ao invés do real. O fato do estudo ter sido realizado via internet trouxe inovações na meto- 
dologia da pesquisa científica usualmente utilizada com adolescentes, tornando-a mais atrativa, entretanto, limita sua utilização às escolas com laboratório de informática operante. Como essa situação é mais incomum em escolas públicas, pode haver viés na amostra estratificada por escola pública/privada.

Apesar dessas limitações, pode-se reiterar que a produção e consumo de alimentos regionais deve ser estimulada e valorizada, já que o Brasil possui uma extensa variedade desse tipo de alimento, nutritivo e de fácil acesso. Essa é uma forma viável de promoção da alimentação saudável e de garantia da segurança alimentar e nutricional. Ressalta-se, ainda, a necessidade da realização de outros estudos que investiguem o consumo de alimentos regionais, uma vez que a literatura é limitada quanto a esse tema e sua relação com a segurança alimentar e nutricional no contexto do DHAA.

\section{A GRADECIMENTOS}

Ao Conselho Nacional de Desenvolvimento Científico e Tecnológico pelo financiamento da pesquisa "Mapeamento da cultura alimentar da população adolescente nas capitais brasileiras e Distrito Federal" (Processo no 559384/2010-6).

\section{COLABORADORES}

SEAC COELHO e MB GUBERT contribuíram na concepção e desenho do estudo, análise de dados e redação final.

\section{REFERÊ NCIAS}

1. Vitolo MR. Hábitos alimentares na adolescência. In: Vitolo MR. Nutrição: da gestação ao envelhecimento. Rio de Janeiro: Rubio; 2008. p.291-7.

2. Instituto Brasileiro de Geografia e Estatística. Pesquisa de Orçamentos Familiares (POF) 2008-2009: análise do consumo alimentar pessoal no Brasil. Rio de Janeiro: IBGE; 2010.

3. Levy RB, Castro IR, Cardoso LO, Tavares LF, Sardinha LMV, Gomes SF, et al. Consumo e comportamento alimentar entre adolescentes brasileiros: Pesquisa Nacional de Saúde do Escolar (PeNSE), 2009. Ciênc Saúde Colet. 2010; 15(Supl. 2):3085-97.

4. Souza AM, Pereira RA, Yokoo EM, Levy RB, Sichieri R. Alimentos mais consumidos no Brasil: Inquérito Nacional de Alimentação 2008-2009. Rev Saúde Pública. 2013; 47(1 Supl.):190S-9S.

5. Burity $V$, Franceschini $T$, Valente F. Segurança alimentar e nutricional e o direito humano à alimentação adequada. In: Direito humano à alimentação adequada no contexto da segurança alimentar e nutricional. Brasília: ABRANDH; 2010 [acesso 2014 abr 3]. Disponível em: http://abrandh.org.br/ download/20101101121244.pdf

6. Kac G, Velásquez-Melendez G, Schlüssel MM, Segall-Côrrea AM, Silva AA, Pérez-Escamilla R. Severe food insecurity is associated with obesity among Brazilian adolescent females. Public Health Nutr. 2012; 15(10):1854-60.

7. Pérez-Escamilla R, Vianna RPT. Food insecurity and the behavioral and intellectual development of children: A review of the evidence. J Appl Res Child. 2012; 3(1).

8. Brasil. Ministério da Saúde. Guia alimentar para a população brasileira. Brasília: Ministério da Saúde; 2008.

9. Freitas MCS, Pena PGL. Dietética e cultura. In: Taddei JA, Lang RMF, Longo-Silva G, Toloni MHA. Nutrição em saúde pública. Rio de Janeiro: Rubio; 2011.

10. Ginani VC. Avaliação da qualidade nutricional, sensorial e cultural de cardápios populares [doutorado]. Brasília: Universidade de Brasília; 2011.

11. Bickel GW, Nord M, Price C, Hamilton W, Cook J. Measuring food security in the United States. Guide to measuring household food security. Revised 2000. Alexandria: United States Department of Agriculture; 2000 [cited 2013 Dec 20]. Available from: http://www.fns.usda.gov/fsec/files/ fsguide.pdf

12. Coelho SEAC. Consumo de alimentos regionais e situação de (in) segurança alimentar e nutricional entre adolescentes brasileiros [mestrado]. Universidade de Brasília; 2014.

13. Instituto Brasileiro de Geografia e Estatística. Pesquisa Nacional de Saúde do Escolar - 2012. Rio de Janeiro: IBGE; 2013.

14. Instituto Brasileiro de Geografia e Estatística. Censo demográfico 2010: educação e deslocamento/ resultados da amostra. Rio de Janeiro: IBGE; 2010.

15. Currie C, Molcho M, Boyce W, Holstein B, Torsheim $T$, Richter M. Researching health inequalities in adolescents: The development of the Health Behaviour in School-Aged Children (HBSC) family affluence scale. Soc Sci Med. 2008 [cited 2014 Apr 7]; 
66(6):1429-36. Available from: http://ac.els-cdn. com/S0277953607006077/1-s2.0-S0277953607 00 6077-main.pdf?_tid =f0fa24a4-b73c-11e2-8eb e-00000aab0f6b\&acdnat $=1367948494 \_a 30$ babee74919a9cf 3086ea8f12022f2

16. Oliveira ISV, Silva MVB, Siqueira LBO. Determinantes do desempenho dos estudantes no vestibular da Universidade Federal da Paraíba. Econ Desenvolv. 2008; 7(2):286-320.

17. Instituto Brasileiro de Geografia e Estatística. Pesquisa Nacional por Amostra de Domicílios - PNAD: segurança alimentar 2004/2009. Rio de Janeiro: IBGE; 2010.

18. Martins MC. Intervenção educativa para utilização de alimentos regionais por famílias de pré-escolares [doutorado]. Fortaleza: Universidade Federal do Ceará; 2010.

19. Brasil. Ministério da Saúde. Alimentos regionias brasileiros. Série Comunicação e Educação em Saúde, n² 21. Brasília: Ministério da Saúde; 2002.

20. Jaime PC, Figueiredo IC, Moura EC, Malta DC. Fatores associados ao consumo de frutas e hortaliças no Brasil, 2006. Rev Saúde Pública. 2009; 43(Supl. 2):57-64.

21. Brasil. Ministério do Desenvolvimento Social e Combate à Fome. Bancos de Alimentos - Institucional. Brasília: Ministério do Desenvolvimento Social e Combate à Fome. [acesso 2014 mar 7]. Disponível em: http://www.mds.gov.br/falemds/perguntasfrequentes/seguranca-alimentar-e-nutricional/redede-equipamentos/banco-de-alimentos/banco-dealimentos-institucional

22. Brasil. Fundo Nacional de Desenvolvimento da Educação. Resolução CD n 32, de 10 de agosto de 2006. Estabelece as normas para a execução do Programa Nacional de Alimentação Escolar. Diário Oficial da União. 200625 ago.

23. Chaves LG, Mendes PNR, Brito RR, Botelho RBA. O programa nacional de alimentação escolar como promotor de hábitos alimentares regionais. Rev
Nutr. 2009; 22(6):857-66. http://dx.doi.org/10.15 90/S1415-52732009000600007

24. Nunes MMA, Figueiroa JN, Alves JGB. Excesso de peso, atividade física e hábitos alimentares entre adolescentes de diferentes classes econômicas em Campina Grande (PB). Rev Assoc Med Bras. 2007; 53(2):130-4.

25. Toral N, Conti MA, Slater B. A alimentação saudável na ótica dos adolescentes: percepções e barreiras à sua implementação e características esperadas em materiais educativos. Cad Saúde Pública. 2009; 25(11):2386-94.

26. Santos JS, Costa COM, Nascimento Sobrinho CL, Silva MCM, Souza KEP, Melo BO. Perfil antropométrico e consumo alimentar de adolescentes de Teixeira de Freitas - Bahia. Rev Nutr. 2005; 18(5):623-32. http://dx.doi.org/10.1590/\$1415-52 732005000500005

27. Veiga GV, Sichieri R. Correlation in food intake between parents and adolescents depends on socioeconomic level. Nutr Res. 2006 [acesso 2014 abr 1]; 26(10):517-23. Disponível em: http://www. sciencedirect.com/science/article/pii/S0271531706 00193X

28. Daniel JMP, Cravo VZ. Olhares antropológicos sobre a alimentação. In: Canesqui AM, Garcia RWD, organizadores. Antropologia e nutrição: um diálogo possível. Rio de Janeiro: Fiocruz; 2005.

29. Jomori MM, Proença RPC, Calvo MCM. Determinantes de escolha alimentar. Rev Nutr. 2008; 21(1):63-73. http://dx.doi.org/10.1590/\$1415-52 732008000100007

30. Estima CCP, Philippi ST, Araki EL, Leal GVS, Martinez MF, Alvarenga MS. Consumo de bebidas e refrigerantes por adolescentes de uma escola pública. Rev Paul Pediatr. 2011; 29(1):41-5.

Recebido: abril 30, 2014 Versão final: maio 5, 2015 Aprovado: maio 21, 2015 
